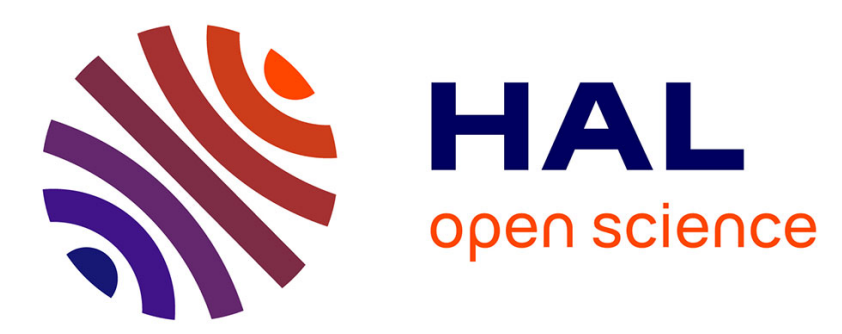

\title{
Mesure par thermographie infrarouge de l'émissivité de matériaux bons conducteurs de la chaleur. Influence de l'état de surface, de l'oxdation et de la température
}

\author{
C. Martin, Pierre Fauchais
}

\section{- To cite this version:}

C. Martin, Pierre Fauchais. Mesure par thermographie infrarouge de l'émissivité de matériaux bons conducteurs de la chaleur. Influence de l'état de surface, de l'oxdation et de la température. Revue de Physique Appliquée, 1980, 15 (9), pp.1469-1478. 10.1051/rphysap:019800015090146900 . jpa00244871

\section{HAL Id: jpa-00244871 \\ https://hal.science/jpa-00244871}

Submitted on 1 Jan 1980

HAL is a multi-disciplinary open access archive for the deposit and dissemination of scientific research documents, whether they are published or not. The documents may come from teaching and research institutions in France or abroad, or from public or private research centers.
L'archive ouverte pluridisciplinaire HAL, est destinée au dépôt et à la diffusion de documents scientifiques de niveau recherche, publiés ou non, émanant des établissements d'enseignement et de recherche français ou étrangers, des laboratoires publics ou privés. 


\title{
Mesure par thermographie infrarouge de l'émissivité de matériaux bons conducteurs de la chaleur. Influence de l'état de surface, de l'oxydation et de la température
}

\author{
C. Martin et P. Fauchais \\ Laboratoire de Thermodynamique, Centre Céramique (ERA 539) de l'Université de Limoges, \\ 123, rue Albert-Thomas, 87060 Limoges Cedex, France \\ (Reçu le $1^{\mathrm{er}}$ octobre 1979, révisé le 2 juin 1980, accepté le 9 juin 1980)
}

\begin{abstract}
Résumé. - La thermographie infrarouge est basée sur l'utilisation d'un pyromètre à balayage optico-mécanique qui fonctionne dans un intervalle de longueur d'onde imposé par son détecteur et son optique. Par exemple pour la caméra A.G.A. utilisée le domaine de longueur d'onde est compris entre 2,0 et 5,6 $\mu \mathrm{m}$.

Cet appareil permet de mesurer des températures de surface à condition de connaître leur émissivité dans ce domaine de longueur d'onde.

Or la littérature ne fournit pas les valeurs de ces émissivités particulières qui ne sont ni monochromatiques ni totales. Dans ce travail, nous avons mesuré par thermographie infrarouge les émissivités de métaux courants. Nous avons étudié les variations de ces facteurs en fonction de l'état de surface, de l'oxydation et de la température et nous avons comparé ces variations, mais non les valeurs absolues, avec celles des émissivités monochromatiques.
\end{abstract}

\begin{abstract}
Infrared thermography is based on the utilization of an optico-mechanical scanning pyrometer working in a wave length band imposed by the detector and the nature of optical components. For example, for the camera A.G.A. utilized, the field of wavelength is included between 2.0 and $5.6 \mu \mathrm{m}$.

This apparatus allows us to measure the surface temperature provided that we know their emissivity over this wavelength range.

But the literature does not give the values of these particular emissivities that are neither monochromatic nor total. In this paper, we have measured by meansol an infrared camera the emissivities of common metals. We have studied the variations of these factors in terms of the effect of surface conditions, of oxidation and the temperature and we have compared these variations, but not the absolue values, with these of monochromatic emissivities.
\end{abstract}

1. Introduction. - Une caméra de thermographie infrarouge est un pyromètre à balayage opticomécanique qui fournit en un temps bref (déterminé par la vitesse du balayage mécanique de la scène, soit 25 images/s) une image visible (noir et blanc) de la surface analysée et dont le degré de noircissement est proportionnel au flux thermique émis par chacun de ces points.

Le flux est fonction d'un grand nombre de paramètres, il dépend non seulement de la température de la surface examinée mais aussi de sa nature, de son état de surface, de sa forme géométrique, de l'angle et de la distance d'observation, de la transparence du milieu séparant l'objet émetteur du détecteur et enfin de la géométrie de l'optique de la caméra. Aussi, l'image thermique obtenue n'apporte aucun renseignement précis quant aux températures réelles de surface sauf dans quelques cas bien particuliers (corps noir plan par exemple).

Il est cependant possible de comparer les flux rayonnés par deux surfaces planes dans le domaine de longueur d'onde $\Delta \lambda=\lambda_{2}-\lambda_{1} \quad\left(\lambda_{1}=2,0 \mu \mathrm{m}\right.$, $\lambda_{2}=5,6 \mu \mathrm{m}$ ) imposé par le détecteur de la caméra et dans certaines conditions, en prenant l'une des surfaces comme référence, de déterminer la température de l'autre si son émissivité est connue [12].

Mais si la littérature fournit beaucoup de données sur les émissivités, elles sont pratiquement inexploitables car en général relatives à des corps purs, placés dans des conditions bien précises d'environnement : vide ou atmosphère contrôlée et pour des états de surface trop souvent mal définis. De plus, elles concernent soit des grandeurs monochromatiques soit des grandeurs totales. Or l'émissivité qui nous intéresse est relative à une bande de longueur d'onde bien déterminée et ne peut être considérée comme absolument monochromatique puisque son degré de monochromatisme, défini par $\mu=\log \lambda_{2} / \lambda_{1}[1]$ est supérieur à l'unité.

Nous appelons cette grandeur émissivité mono- 
chromatique à large bande notée d'une manière générale $\varepsilon(\Delta \lambda, \theta, \varphi, T)$ où $\theta$ et $\varphi$ sont respectivement la colatitude et l'azimut de la direction d'émission; ces angles sont définis par rapport à la normale ON à la surface et à une direction origine $O X$ située dans le plan tangent à la surface en $0 ; T$ est la température du corps émetteur.

Dans ce travail cependant, nous ne mesurons que des émissivités normales, c'est-à-dire pour $\theta=\varphi=0$ et nous écrirons cette grandeur $\varepsilon(\Delta \hat{\lambda}, T)$. Nous avons mis au point une méthode de mesure de ces émissivités par thermographie infrarouge en nous intéressant à des surfaces naturelles puisque la vocation d'un tel appareil est de réaliser des mesures rapides in situ. Nous avons étudié, pour des corps bons conducteurs de la chaleur, la variation de ces émissivités avec la température, la rugosité et l'oxydation.

Comme ces émissivités monochromatiques à larges bandes (notée émissivités M.L.B.) sont définies d'une manière particulière et s'adressent à des métaux soumis à une oxydation naturelle nous n'avons pas comparé nos résultats aux valeurs absolues des émissivités monochromatiques, mais leurs variations ont été confrontées à celles données pour les émissivités monochromatiques.

2. La mesure des émissivités M.L.B. - 2.1 LA THERMOGRAPHIE INFRAROUGE. - La thermographie infrarouge est un processus qui donne «la représentation de la distribution des flux superficiels d'un corps, obtenue à partir du rayonnement électromagnétique émis par les objets ".

Nous avons utilisé une caméra type 750 de la Société Suédoise A.G.A. dont nous ne rappelons ici que le principe de la fonction isoflux. Une description plus détaillée peut être trouvée dans la littérature en particulier chez [13].

L'appareil est constitué de deux unités, une unité de prise de vues, la caméra proprement dite et une unité de visualisation qui est un oscilloscope sur l'écran duquel nous observons l'image thermique de la scène analysée. Celle-ci se présente sous forme d'un dégradé de teinte allant du blanc pour les parties chaudes au noir pour les parties froides. Ce dégradé de teinte correspond à des niveaux d'énergie différents. Sur l'oscilloscope un potentiomètre, couplé à un index de lecture, permet de visualiser sur l'écran les zones de même luminance, donc de repérer les surfaces qui émettent le même flux.

Lorsque nous utilisons cette correspondance entre le flux émis par une surface et l'indication de l'index de lecture, nous disons que nous utilisons la fonction isoflux.

2.2 Principe DE LA Mesure. - Nous exploitons essentiellement la propriété de la fonction isoflux de notre appareillage. Nous comparons les flux émis par l'échantillon de mesure et un corps de référence; lorsque ces flux sont égaux, l'émissivité de l'échantillon est donnée par la relation $[13,16]$.

$$
\varepsilon\left(\Delta \hat{\imath}, T_{1}\right)=\frac{T_{2}^{4}\left(z\left(x_{2,2}\right)-z\left(x_{1,2}\right)\right)-T_{\mathrm{a}}^{4}\left(z\left(x_{2, \mathrm{a}}\right)-z\left(x_{1, \mathrm{a}}\right)\right)}{T_{1}^{4}\left(z\left(x_{2,1}\right)-z\left(x_{1,1}\right)\right)-T_{\mathrm{a}}^{4}\left(z\left(x_{2, \mathrm{a}}\right)-z\left(x_{1, \mathrm{a}}\right)\right)} x \varepsilon\left(\Delta i, T_{2}\right)
$$

Les indices 1,2 et a sont respectivement relatifs à l'échantillon, au corps de référence et à l'ambiance, celle-ci pouvant être considérée comme une enceinte fermée qui rayonne vers l'échantillon comme un corps noir [11]. $T$ désigne la température $(K)$ et $z(x)$ :

$$
z(x)=\frac{\int_{0}^{\lambda} L^{0}(\lambda, T) \mathrm{d} \lambda}{\int_{0}^{\infty} L^{0}(\lambda, T) \mathrm{d} \lambda}
$$

est une fonction qui se rapporte au corps noir (exposant 0 ) et se trouve tabulée dans la littérature [8]. Ici :

$$
z\left(x_{2,1}\right)=z\left(\frac{\lambda_{2}}{\lambda_{\mathrm{m}}}\right) \quad \text { avec } \quad \lambda_{\mathrm{m}} T_{1}=2897,8 \mu \mathrm{m} . \mathrm{K}[10]
$$

et

$z\left(x_{1,1}\right)=z\left(\frac{\lambda_{1}}{\lambda_{\mathrm{m}}}\right)$ $\hat{\lambda}_{1}$ et $\hat{\lambda}_{2}$ sont les bornes du domaine spectral de notre détecteur.

Si la référence est le corps noir, il suffit, dans la relation précédente, d'écrire :

$$
\varepsilon\left(\Delta \hat{\lambda}, T_{2}\right)=1 \text {. }
$$

2.3 Les ÉChantillons. - Pour notre étude, nous avons choisi trois métaux ordinaires, le cuivre, pur à $99,6 / 100$, car c'est un métal qui possède une des meilleures conductivités thermiques aux températures qui nous intéressent ; l'acier, connu commercialement sous l'appelation XC38F $(0,32 / 100$ de $\mathrm{C} ; 0,50 / 100$ de $\mathrm{Mn}$ et $0,4 / 100$ de $S$ ) qui est très répandu et enfin l'aluminium, pur à 99,4/100 qui est aussi très utilisé mais qui a des propriétés émissives particulières, se recouvrant très rapidement d'alumine à la température ambiante. Pour chacun de ces échantillons nous avons réalisé trois états de surface bien déterminés en les usinant avec un étau limeur qui, par son outil de pente d'affutage de $80^{\circ}$, trace des sillons parallèles sur leur surface avec des pas respectifs de $0,1 \mathrm{~mm}, 0,2 \mathrm{~mm}$ et $0,5 \mathrm{~mm}$. 
Tableau I. - Caractéristiques de l'état de surface des matériaux.

[Characteristic of the surface conditions of materials.]

\begin{tabular}{|c|c|c|c|c|}
\hline MATERIAUX & $\begin{array}{c}\text { Distance des } \\
\text { stries } \\
(\mathrm{mm}) \\
\end{array}$ & $\sigma(\mu \boldsymbol{N})$ & $R_{d}(\mu m)$ & $\frac{\sigma_{x}}{\lambda}$ \\
\hline \multirow{3}{*}{$x C 38 F$} & $1 / 10$ & 10,1 & 9 & 1,66 \\
\hline & $2 / 10$ & 10.8 & 9 & 2,84 \\
\hline & $5 / 10$ & 22,5 & 19 & 5,92 \\
\hline \multirow{3}{*}{$\mathrm{Cu}$} & $1 / 10$ & 12,2 & 10,1 & 3,21 \\
\hline & $2 / 10$ & 15 & 13,2 & 3,94 \\
\hline & $5 / 10$ & 24 & 22 & 6,32 \\
\hline \multirow{3}{*}{$A 1$} & $1 / 10$ & 7,8 & 6,6 & 2,05 \\
\hline & $2 / 10$ & 21,5 & 18,8 & 5,65 \\
\hline & $5 / 10$ & 225 & $>25$ & \\
\hline
\end{tabular}

L'état de surface peut être défini à partir de fonctions statistiques, et le critère ordinairement choisi est le rapport $\sigma_{z} / \lambda$ de la rugosité à la longueur d'onde des radiations, où $\sigma_{z}$ est l'écart-type sur la hauteur des aspérités défini par

$$
\sigma_{z}=\int_{-\infty}^{+\infty}(z-\bar{z})^{2} f(z) \mathrm{d} z
$$

où $z$ est la cote d'un point de surface par rapport à un plan de référence, $\bar{z}$ la moyenne des cotes par rapport à ce plan de référence et $f(z)$ la fonction de distribution des hauteurs.

Si $\sigma_{z}$ est voisin de $\lambda$, deux fonctions statistiques suffisent pour caractériser l'état de surface [2]. La deuxième fonction utilisée est en général le moment d'ordre 1 de la série considérée, c'est-à-dire la rugosité moyenne arithmétique définie par :

$$
R_{\mathrm{a}}=\int_{-\infty}^{+\infty}(z-\bar{z}) f(z) \mathrm{d} z .
$$

Dans le tableau I nous indiquons les valeurs de $\sigma_{z}, R_{\mathrm{a}}$ et $\sigma_{z} / \lambda$ correspondant aux échantillons que nous avons utilisés.

3. Montage expérimental. - Nous avons utilisé des échantillons sous forme de pastilles de $23 \mathrm{~mm}$ de diamètre et de 3 à $4 \mathrm{~mm}$ d'épaisseur (Fig. 1).

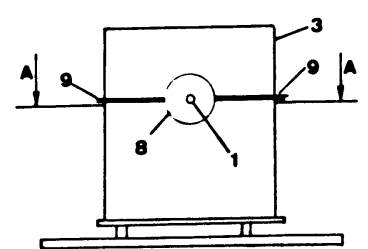

VUE DE FACE (6:1/10)

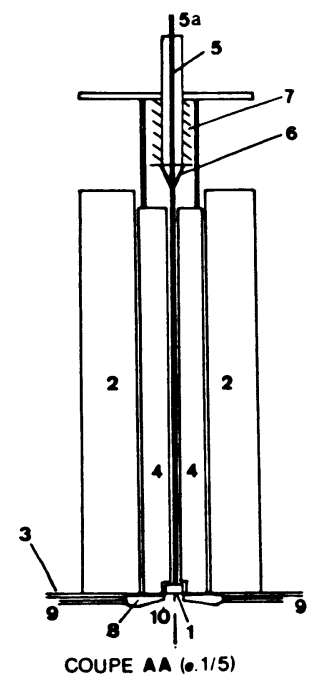

Fig. 1. - 1. Echantillon. 2. Four. 3. Protection en amiante. 4. Cylindre réfractaire. 5. Thermocoaxe. 5a. Vers l'enregistreur. 6. Pince de serrage du thermocoaxe. 7. Ressort de pression. 8. Ecran refroidi. 9. Circulation d'eau. 10. Axe de visée de la caméra.

[1. Sample. 2. Furnace. 3. Safety device in asbestos. 4. Refractory cylinder. 5. Thermocouple. 6. Champ for thermocouple. 7. Compression-springer. 8. Cooled screen. 9. Circulation of water. 10. Axis of aim of camera.]

Elles sont serties à l'aide d'un ciment réfractaire, qui permet un bon contact thermique, dans une cavité située à l'extrémité d'un cylindre en acier 
réfractaire de $30 \mathrm{~mm}$ de diamètre et $150 \mathrm{~mm}$ de longueur. L'ensemble est plongé dans un four tubulaire de $31 \mathrm{~mm}$ de diamètre, régulé et qui peut atteindre $1650 \mathrm{~K}$. Le positionnement de la pastille s'effectue de manière à ce que sa surface visée soit dans le plan de la surface circulaire du cylindre support et de l'ouverture du four. Ce plan, également confondu avec celui de l'ouverture du corps noir de référence, est perpendiculaire à l'axe de visée de la caméra. La pastille est percée d'un trou borgne central de $1,5 \mathrm{~mm}$ de diamètre dont le fond arrive à 0,1 ou $0,2 \mathrm{~mm}$ de sa surface. Un thermocouple traverse tout le four (Fig. 1, (5)) et est enfoncé en force dans ce trou borgne.

Nous avons montré [16] qu'un tel montage n'entraîne pas d'incertitude relative supérieure à 0,015 sur la mesure des températures de surface.

Les surfaces de l'échantillon et du corps noir sont fixes et dans un même plan vertical; elles sont successivement visualisées par la caméra qui se déplace suivant une direction parallèle au plan précédent. Nous mesurons ainsi l'émissivité normale c'est-à-dire $\varepsilon(\Delta \lambda, T)$.

Nous avons vérifié expérimentalement que la distance du corps noir à la caméra ne devait pas être supérieure à $3,5 \mathrm{~m}$ [12] pour que son image à travers le système optique de la caméra recouvre entièrement la surface sensible du détecteur. Aussi, nous avons effectué nos visées à une distance inférieure ou égale à $3 \mathrm{~m}$. De plus, la comparaison des flux s'est toujours effectuée lorsque les images de l'échantillon et du corps noir sont placées sur la même zone de l'écran pour éviter ses défauts d'homogénéité.

4. Procédure expérimentale. - Nous pouvons comparer les flux de l'échantillon et du corps noir soit à l'égalité, soit en faisant intervenir un corps noir fictif.

La première méthode consiste d'abord à régler la température de l'échantillon à la valeur choisie puis à amener, à l'aide de la fonction isoflux, la surimpression en blanc sur l'image de sa surface et enfin à régler progressivement la température du corps noir de manière à ce que la surimpression en blanc affecte également l'image de sa surface. Lorsqu'un tel équilibre est atteint nous relevons les températures de l'échantillon, du corps noir et de l'ambiance la relation (1) nous donne l'émissivité cherchée.

Cette méthode est longue et il est préférable quelquefois d'utiliser la méthode du corps noir fictif qui est bien plus rapide mais un peu moins précise. Nous réglons toujours la température de l'échantillon à la valeur choisie mais cette fois, celle du corps noir à une valeur voisine. A l'équilibre, nous amenons successivement la surimpression en blanc sur la pastille et sur le corps noir, en agissant sur le potentiomètre de la fonction isoflux; pour chacun de ces réglages, nous lisons l'indication de l'index et, connaissant la température du corps noir, la courbe d'étalonnage de l'appareil nous permet de déterminer la température d'un corps noir fictif qui émet le même flux que la pastille [12]. Nous sommes alors ramenés au problème précédent.

5. Résultats. - Le volume des calculs nous a conduit à élaborer un programme dont le traitement est réalisé par un ordinateur CII (Mitra 15) qui nous donne les valeurs des émissivités et les droites de régression par rapport à la température. Sur les graphiques que nous présentons nous faisons figurer l'équation de ces droites ainsi que le coefficient de corrélation (noté Correl) et l'incertitude moyenne (notée $\overline{\Delta \varepsilon})$.

Nous avons voulu déterminer les émissivités de ces matériaux dans des conditions réelles d'utilisation, c'est-à-dire sans aucune précaution particulière d'environnement. Nous avons étudier les émissivités normales en fonction de la température, de l'oxydation et de la rugosité. Les mesures ont tout d'abord été réalisées pour des températures croissantes. Puis, après avoir laissé les pastilles pendant $72 \mathrm{~h}$ à une température voisine de $900 \mathrm{~K}$ nous avons de nouveau effectué des mesures mais pour des températures décroissantes. Nous rappelons que la surface visée est striée et se trouve dans un plan vertical perpendiculaire à l'axe de visée. Nous avons fait tourner l'échantillon autour de cet axe et vérifié que l'émission normale de cette surface était indépendante de l'orientation des stries par rapport à la direction verticale. Nous avons constaté un comportement émissif différent pour ces trois matériaux.

Dans le domaine de température considéré, nos résultats, en accord avec ceux concernant les émissivités monochromatiques $[5,19]$ nous donnent une loi de variation linéaire de l'émissivité M.L.B. en fonction de la température.

\subsection{VARIATION EN FONCTION DE LA TEMPÉRATURE} et de L'oxydation. - 5.1.1 Aluminium. - Pour l'aluminium le coefficient de température est négatif et reste du même ordre de grandeur, que les mesures soient effectuées en températures croissantes ou en températures décroissantes (Fig. 2). Ce phénomène s'explique par le fait que l'aluminium s'oxyde naturellement à la température ambiante [6]; dans nos conditions expérimentales, la couche d'oxyde ayant une épaisseur voisine de $5 \mu \mathrm{m}$ c'est l'oxyde et non le métal qui rayonne et nous trouvons bien une loi de variation correspondant à celle des corps mauvais conducteurs dont l'émissivité décroit avec la température.

5.1.2 Acier. - Pour l'acier XC38F nous avons étudié plus spécialement l'effet de l'oxydation sur l'échantillon $5 / 10$, en effectuant plusieurs séries de mesures consécutives pour des températures croissantes puis pour des températures décroissantes. 


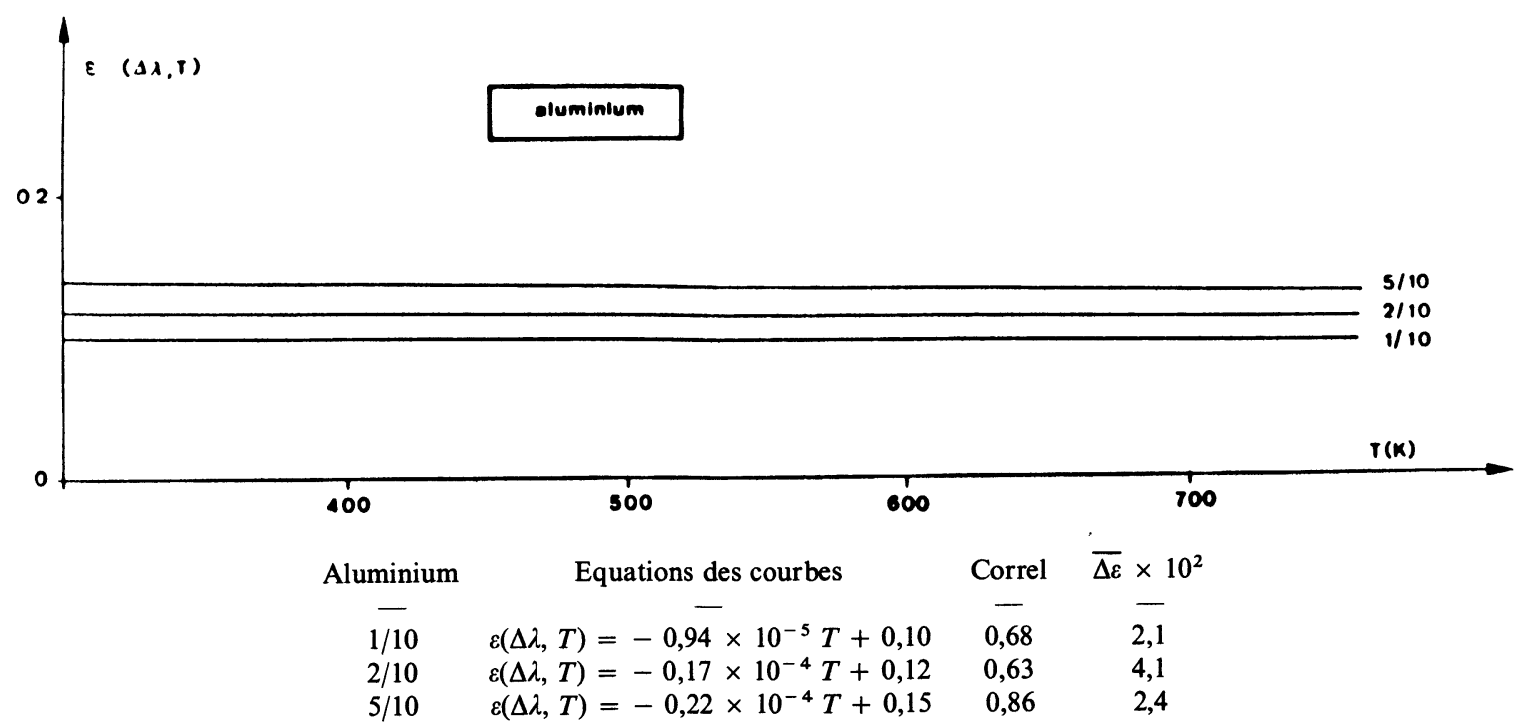

Fig. 2. - Variation de l'émissivité en fonction de la rugosité pour l'aluminium.

[Emissivity variation of aluminium versus roughness.]

Dans la première série nous sommes partis d'un échantillon non oxydé et nous avons fait des mesures jusque vers $800 \mathrm{~K}$. Nous remarquons alors l'existence de deux zones distinctes de variation de part et d'autre de $600 \mathrm{~K}$ (Figs. 3 et 4). Il y a une discontinuité dans la variation de l'émissivité et modification du coefficient de température. Cette discontinuité, ne pouvant être interprétée comme le passage au point de Curie, qui, pour ce matériau, est à $1043 \mathrm{~K}$ [20], est vraisemblablement due à une évolution de l'oxydation.
Ce résultat est confirmé par les séries de mesures suivantes. Nous avons en effet renouvelé ces mesures avec le même échantillon toujours pour des températures croissantes et nous avons obtenu les courbes de la figure 5. Les chiffres arabes, de 1 à 4 placés sur ces courbes, indiquent l'ordre chronologique. Nous constatons que le coefficient de température d'abord positif devient négatif et se stabilise, ce qui montre bien une évolution de l'oxydation en fonction du temps. Nous avons renouvelé ces mesures avec

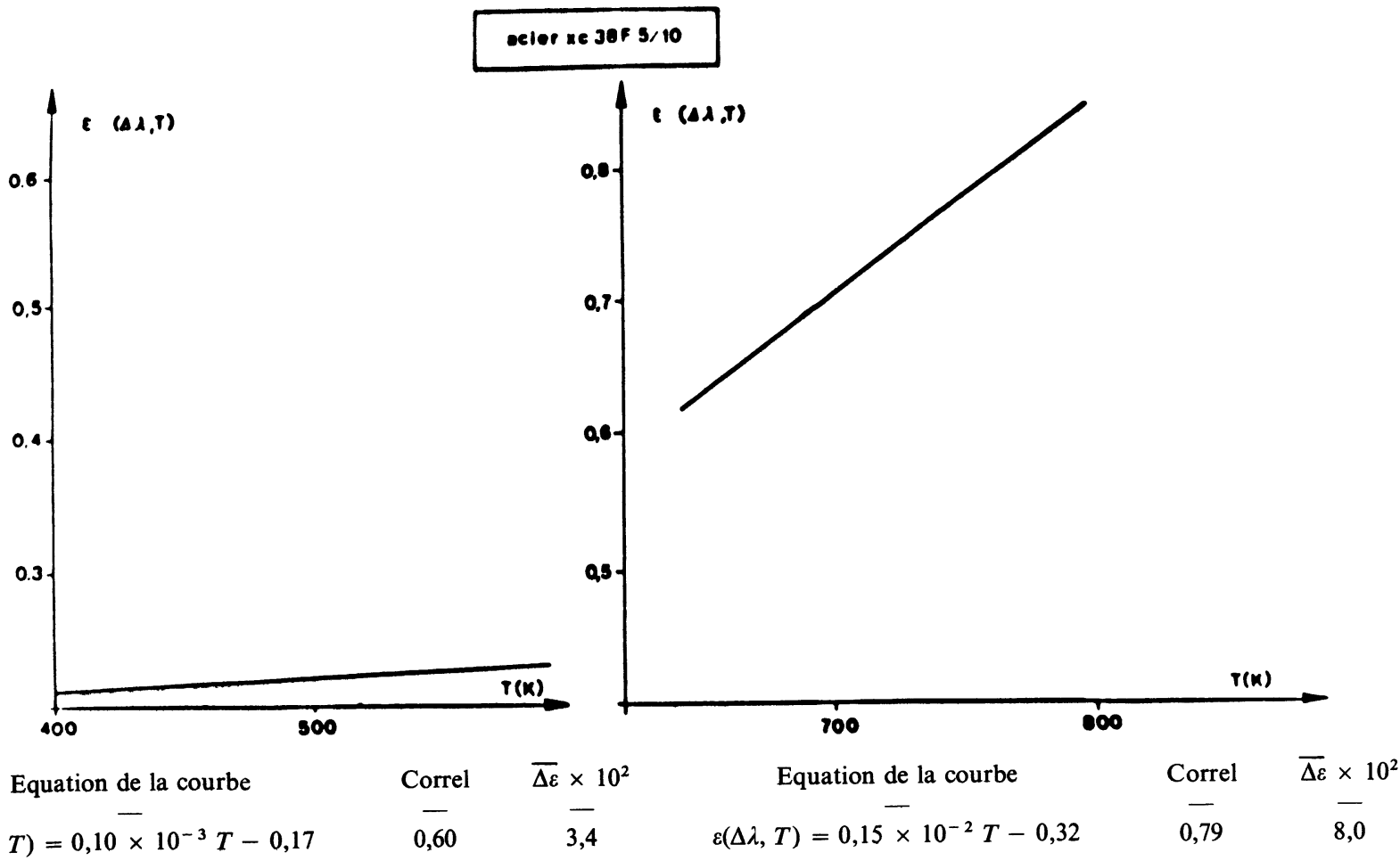

$\varepsilon(\Delta \lambda, T)=0,10 \times 10^{-3} T-0,17 \quad \overline{0}, \quad \overline{3,4}$

Fig. 3. - Variation du coefficient de température pour l'acier.

[Temperature coefficient versus temperature for steel.]
Fig. 4. - Variation du coefficient de température pour l'acier.

[Temperature coefficient versus temperature for steel.] 


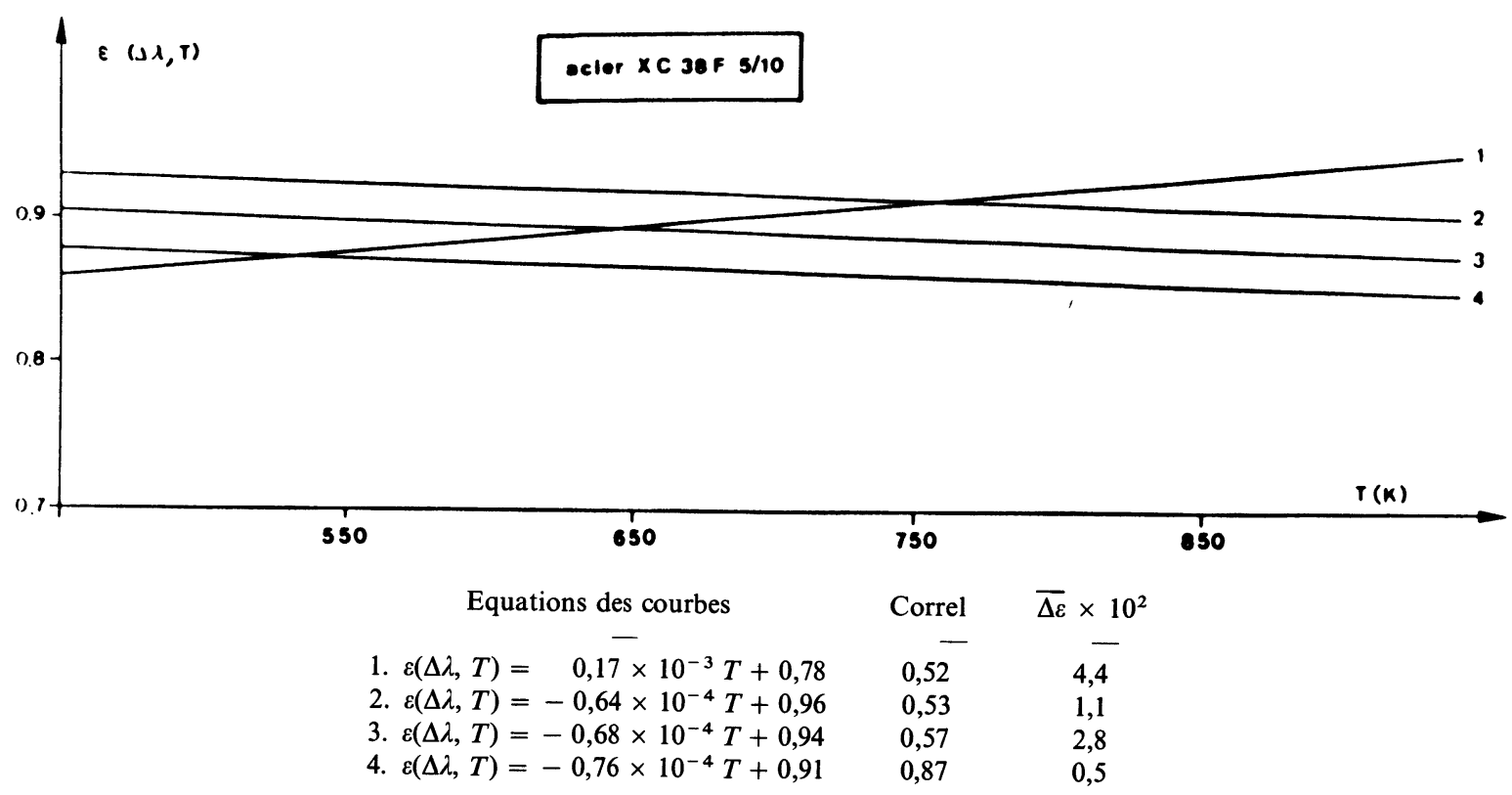

Fig. 5. - Variation de l'émissivité en fonction de l'oxydation pour l'acier.

[Emissivity variation of steel versus oxidation.]

un autre échantillon et pour des températures croissantes, puis décroissantes, après avoir laissé l'échantillon $72 \mathrm{~h}$ à une température voisine de $900 \mathrm{~K}$. Les relations obtenues sont respectivement :

$$
\begin{aligned}
& \varepsilon(\Delta i, T)=-0,15 \times 10^{-4} T+0,98 \\
& \varepsilon(\Delta i, T)=-0,58 \times 10^{-4} T+0,92 .
\end{aligned}
$$

Les courbes de la figure 6 représentent la variation de la pente en fonction de l'oxydation. Les chiffres arabes ont la même signification que plus haut, la courbe 5 correspond à l'équation (5). Nous pouvons donc dire que l'oxydation modifie le caractère émissif du matériau et, assez rapidement, amène le coefficient de température à une valeur constante.

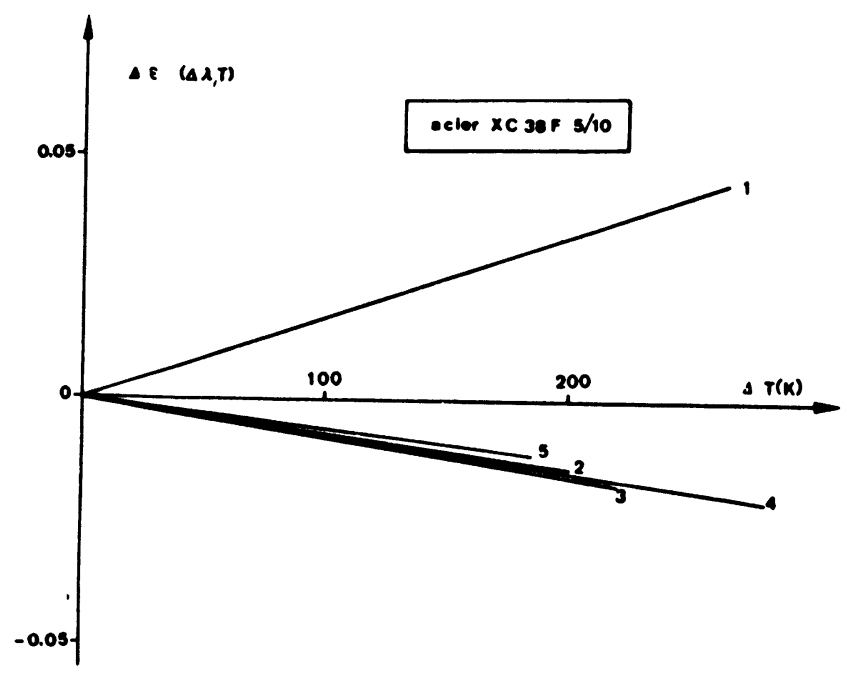

Fig. 6. - Un accroissement de l'émissivité en fonction d'un accroissement de température pour différents états d'oxydation.

[Increment of emissivity versus increment of temperature for different degrees of oxidation.]
L'émissivité de l'acier oxydé a donc aussi le même sens de variation que celui d'un mauvais conducteur ce qui est en accord avec les résultats obtenus par [14] qui précise que $\varepsilon_{i}(\lambda, T)$, décroît avec la température pour les oxydes métalliques. Ce résultat concerne des émissivités monochromatiques mais nous pouvons dire que dans cet intervalle de longueur d'onde, la variation de l'émissivité M.L.B. est la même que celle de l'émissivité monochromatique. Nous avons constaté le même phénomène avec les autres échantillons d'acier $2 / 10$ et $1 / 10$ (Fig. 7) que nous avions laissé $72 \mathrm{~h}$ à $900 \mathrm{~K}$ environ.

5.1.3 Cuivre. - Le cuivre par contre se comporte différemment. Il conserve une émission métallique en dépit de l'oxydation. De plus, pour ce métal, l'oxydation n'est pas un phénomène stable; en effet, vers $900 \mathrm{~K}$ les pastilles se recouvrent d'une mince pellicule noire (oxyde $\mathrm{CuO}$ ) mais lorsque la température diminue cette pellicule s'écaille et disparaît pour laisser une surface présentant un aspect grisâtre ( $\mathrm{CuO}$ en couche mince). Les résultats donnés par les courbes de la figure 8 sont ceux relatifs à cet état. Ceux de la figure 9, avec les équations des courbes correspondantes, représentent pour le cuivre $1 / 10$ l'évolution de $\varepsilon(\Delta i, T)$ en fonction de l'oxydation. Les pastilles à l'aspect grisâtre sont recouvertes d'un film d'oxyde $\mathrm{CuO}$ et le résultat que nous trouvons est en accord avec [3] qui indique que dans l'infrarouge l'épaisseur du film d'oxyde n'a pratiquement pas d'influence sur l'émissivité, c'est-à-dire que c'est le métal et non l'oxyde qui émet comme l'indiquent nos courbes.

5.2 VARIATION EN FONCTION DE LA RUGOSITÉ. Une mesure d'émissivité n'est intéressante que si 


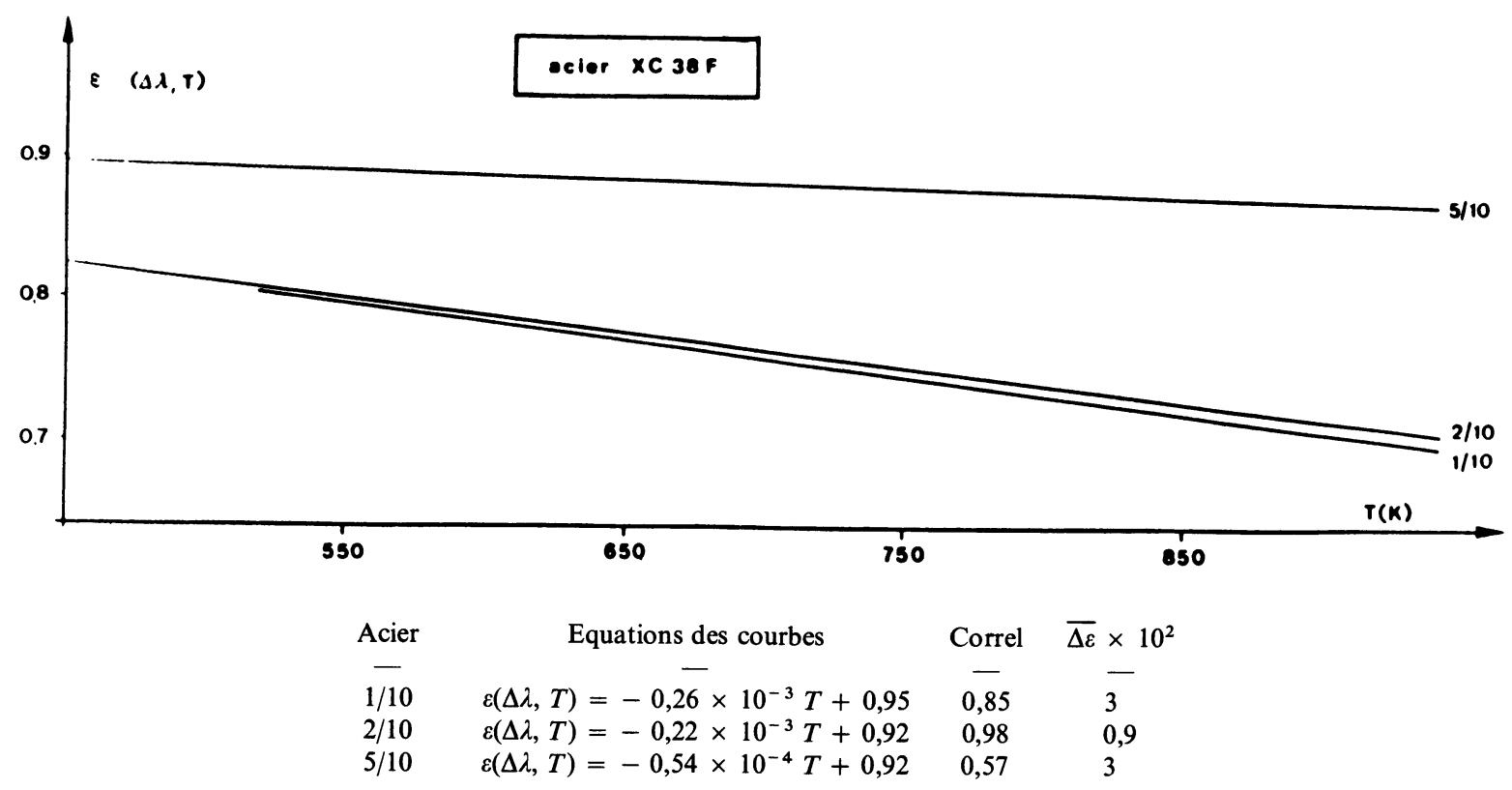

Fig. 7. - Variation de l'émissivité en fonction de la rugosité pour l'acier.

[Emissivity variation of copper versus roughness.]

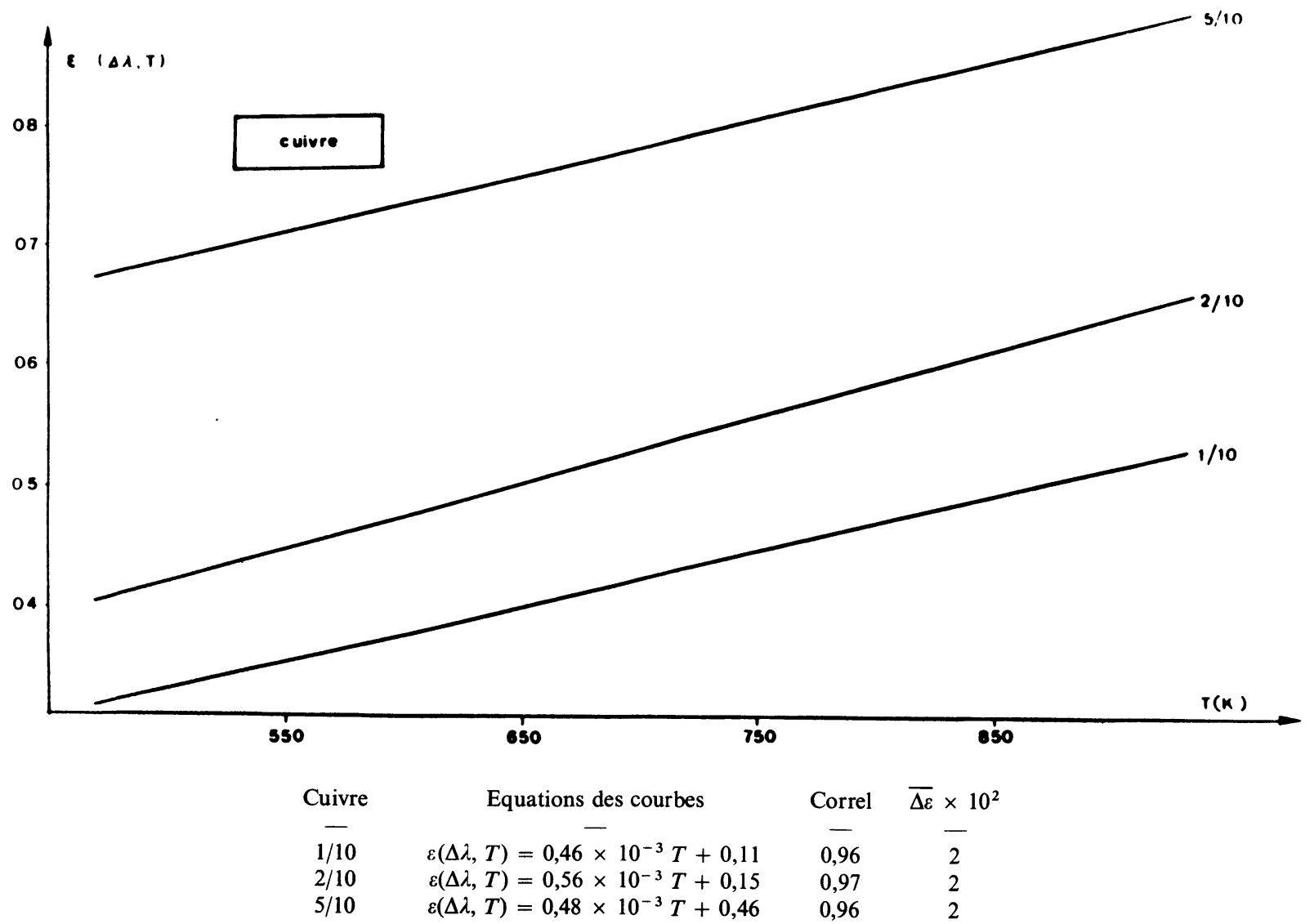

Fig. 8. - Variation de l'émissivité en fonction de la rugosité pour le cuivre.

[Emissivity variation of copper versus roughness.] 


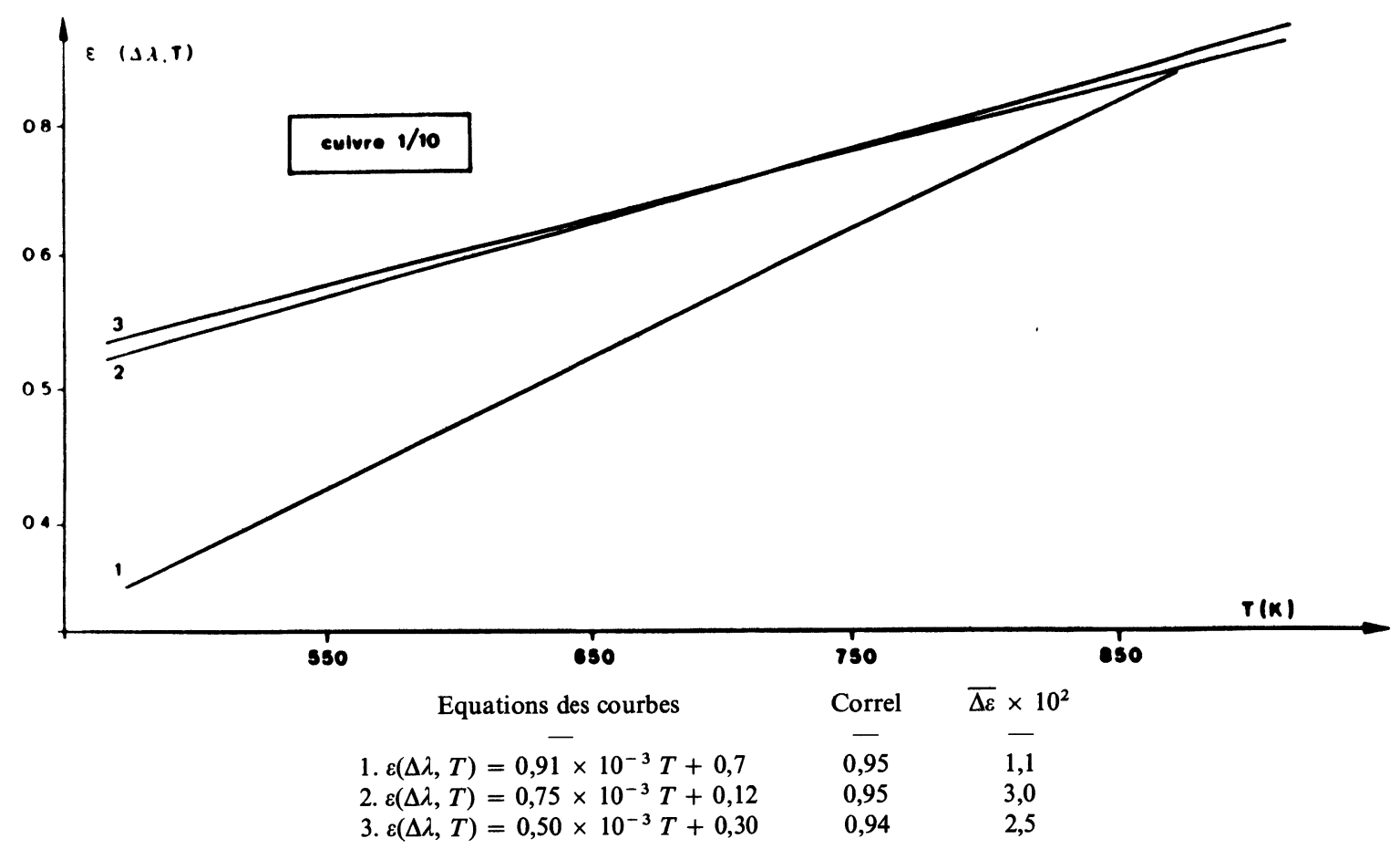

Fig. 9. - Variation de l'émissivité en fonction de l'oxydation.

[Emissivity variation versus oxidation.]

l'état des surfaces émettrices est connu; c'est pourquoi nous avons fait réaliser pour nos échantillons des surfaces à structures bien déterminées que nous analysons à l'aide d'un perthomètre, sur une longueur de $5 \mathrm{~mm}$ suivant une ligne perpendiculaire à l'arête des stries. Nous donnons dans le tableau I les différentes valeurs de $\sigma_{z}$, de $\sigma_{z} / \hat{\lambda}$ et de Ra, puisque $\sigma_{z}$ est voisin de $i$ (nous prenons pour $i$ la valeur moyenne de notre intervalle de longueur d'onde c'est-à-dire $3,8 \mu \mathrm{m}$ ).

Nous voyons que ce rapport $\sigma_{z} / i$ est compris entre 2 et 7 c'est-à-dire que nos surfaces peuvent être considérées comme rugueuses par rapport à la longueur d'onde [9]. Nous constatons que, comme pour les émissivités monochromatiques, l'émissivité que nous mesurons est une fonction croissante de la rugosité. Pour le moment nous ne pouvons établir qu'une loi qualitative entre l'émissivité et la rugosité puisque d'une part, sauf pour le cuivre, nous n'avons étudié que trois types de rugosité pour chaque matériau et d'autre part l'oxydation (les mesures sont faites à l'ambiante) perturbe trop ce phénomène d'émission pour permettre comme le font de nombreux auteurs $[17,4,9]$ de comparer nos facteurs à ceux du métal considéré comme lisse.

Ces auteurs relient la réflectivité monochromatique normale $\mathbf{R}$ d'un matériau à sa rugosité $\sigma_{z}$ par une ręlation de la forme :

$$
\frac{R}{R_{0}}=\exp \left(-k\left(\frac{\sigma_{z}}{i}\right)^{2}\right)
$$

où $R_{0}$ représente la réflectivité monochromatique du matériau considéré parfaitement lisse.
Partant de cette relation nous avons tracé les courbes (Fig. 10) qui donnent, pour le cuivre et pour différentes températures, la variation de $\log R / R_{0}$ en fonction de $\left(\sigma_{z} / \hat{i}\right)^{2}$.

Nous n'avons utilisé que le cuivre car comme nous l'avons vu plus haut, c'est le seul dont l'émission n'est pas trop perturbée par l'oxydation et nous avons pu ainsi déterminer la valeur $R_{0}$ par la relation de Drude [7]

$$
R_{0}=1-\left(0,365\left(\frac{\rho}{i}\right)^{1 / 2}+0,066 \frac{\rho}{i}\right)
$$

où $\rho$ est la résistivité électrique du matériau à la longueur d'onde moyenne de notre intervalle $(3,8 \mu \mathrm{m})$.

Nous constatons sur ces courbes (Fig. 10) que la droite obtenue pour la température la plus basse, c'est-à-dire $450 \mathrm{~K}$, tend à se déformer pour présenter une allure nettement concave pour la température la plus élevée $(850 \mathrm{~K})$.

Ce que nous expliquons par le fait que l'oxydation est de plus en plus prépondérante et donc que la réflectivité du matériau suit de moins en moins bien la loi traduite par l'équation (6).

Nous n'avons pas poussé plus avant cette analyse car il nous importait surtout dans cette première étude de mesurer des émissivités par thermographie infrarouge à partir de la relation (1) que nous avions établie.

6. Conclusions. - Nous n'avons pas trouvé dans la littérature de résultats concernant des émissivités M.L.B., mais les métaux que nous avons étudiés ne présentent pas de pics d'absorption dans le domaine 


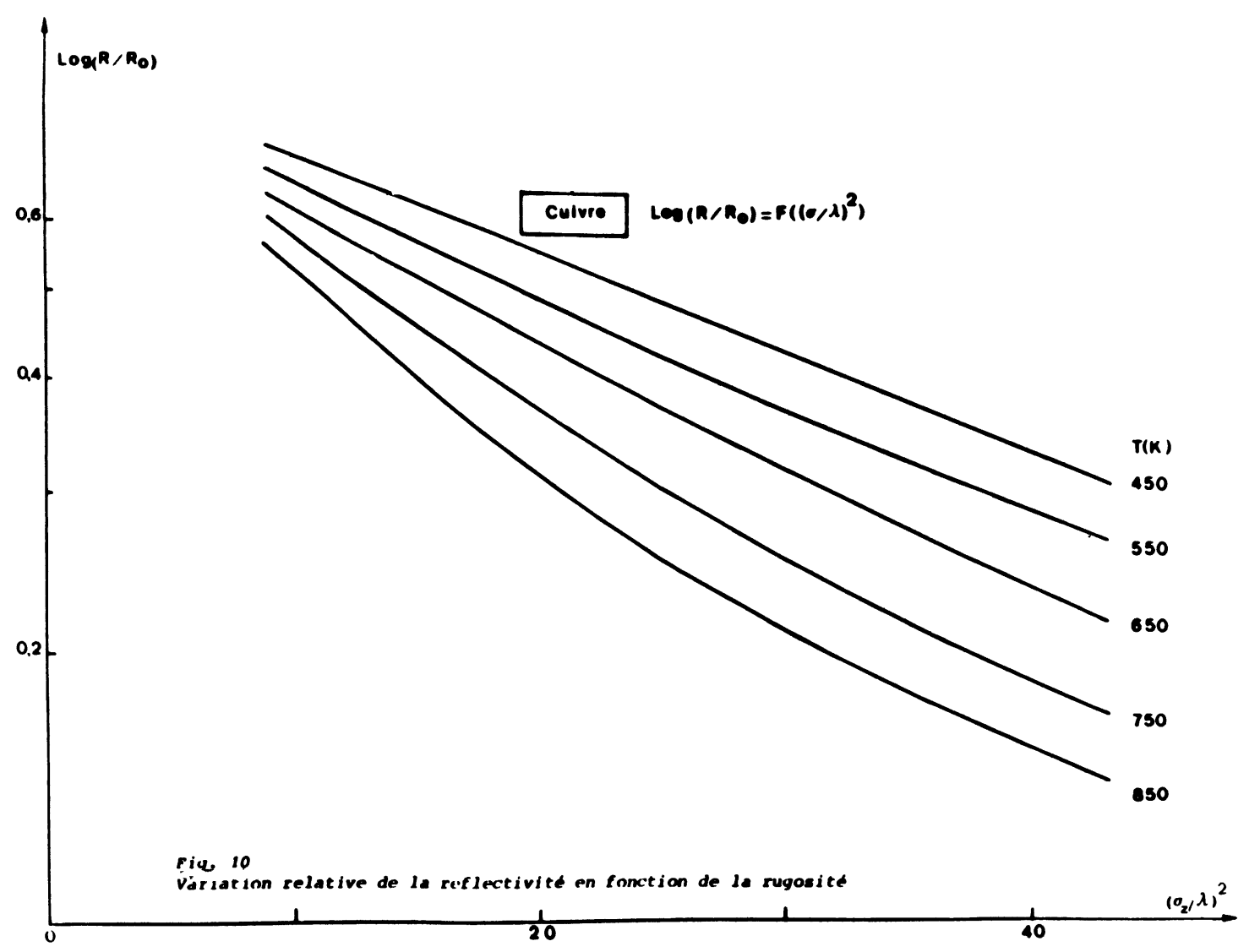

Fig. 10. - Variation relative de la réflectivité en fonction de la rugosité.

[Reflectivity relative variation versus roughness.]

de longueur d'onde étudié, et, de plus, nous sommes loin du point " $X$ » de ces métaux qui se situent aux alentours de $1,2 \mu \mathrm{m}$ [18], aussi nous faisons l'hypothèse que notre $\varepsilon(\Delta \lambda, T)$ est égal à l'émissivité monochromatique moyenne $\bar{\varepsilon}_{\lambda}(\hat{\lambda}, T)$ dans l'intervalle $\Delta \lambda \hat{\lambda}$ considéré.

La relation que nous proposons nous a permis de calculer les émissivités M.L.B. de matériaux bons conducteurs de la chaleur. Il n'était pas question de comparer nos valeurs absolues à celles, monochromatiques, données dans la littérature puisque, d'une part, nos états de surface sont particuliers et, d'autre part, nos échantillons sont oxydés, mais essentiellement de vérifier que les sens d'évolution de l'émissivité M.L.B., en fonction de l'oxydation, de la température et de la rugosité étaient identiques à ceux indiqués pour les émissivités monochromatiques.

Pour une température donnée, l'émissivité M.L.B. croît avec la rugosité et l'oxydation; il faut noter que celle-ci est un phénomène non négligeable qui influence fortement l'émissivité des corps métalliques.
Les métaux que nous avons choisis nous ont permis de mettre en évidence trois types de comportement. Celui de l'aluminium qui déjà oxydé à l'ambiante se comporte toujours comme un mauvais conducteur. Celui de l'acier qui s'oxyde en fonction de la température et, après s'être recouvert d'une pellicule épaisse d'oxyde, possède une émission diélectrique, dont l'état reste stable contrairement au cuivre qui, sous l'effet d'une forte oxydation, se recouvre d'une épaisse couche de $\mathrm{CuO}$ et émet alors comme un diélectrique mais ne reste pas dans cet état. Par contre, pour une oxydation modérée le cuivre se recouvre d'un mince film de $\mathrm{CuO}$ et conserve une émission métallique.

Nous avons enfin pu vérifier que $\varepsilon(\Delta \lambda, T)$ décroît avec la température pour les oxydes métalliques [14], croît avec la température pour le cuivre recouvert d'un oxyde en couche mince [3] et croît également avec la rugosité comme le montre théoriquement Sacadura [15].

\section{Bibliographie}

[1] Bramson, M. A., Infrared radiation (Plenum Press, New York) 1968.

[2] Bennett, H. E., Porteus, J. O., J. Opt. Soc. Am. 51 (1961) $123 / 29$.
[3] BENNETT, H. E., Influence of surface roughness, surface damage and oxide film on emittance. Symposium on thermal radiation of solids, San Francisco, California, 4 au 6 mars 1964, ED. NASA-SP, 55-1965. 
[4] BirkebaK, R. C., Eckert, E. R. G., J. Heat Transf. (1965) 85.

[5] Cabannes, F., J. Physique, 28 (1967) 235/48.

[6] Cabrera, N., Revue de métallurgie XLV (1948) 86/92.

[7] Drude, P., Physik des Athers, Stuttgart 1894, p. 574.

[8] FABRY, C., Introduction générale à la photométrie théorique et expérimentale. Ed. Revue d'Optique, Paris (1927).

[9] Houchens, A. F., Hering, R. G., Radiant Heat transfer for rough surface. Proceedings of the fifth international heat transfer conference. 3-7 Sept. 1974, Keidanrenkaikan building Tokio.

[10] HuETz-Aubert, Définitions et lois fondamentales. Ecole d'été Perpignan, 6-8 sept. 1976

[11] Jatteau, M., Acta Electronica, 12 (1), 1969, 21/62.

[12] Martin, C., Pateyron, B., Fauchais, P., Contribution à la mesure du facteur d'émission normal de métaux et de céramiques par thermographie infrarouge. Applications pratiques à la mesure des températures de surface. Bulletin d'information du G.U.T., Paris, 3 juin 1974, 131/74.
[13] Martin, C., Contribution à l'utilisation de la thermographie infrarouge pour les mesures de flux, de températures et de divers paramètres, thèse ès Sciences, Limoges 1978.

[14] Michaud, M., Facteurs d'émission d'oxydes métalliques et réfractaires à hautes températures, Thèse ès Science, Paris, 1951

[15] Sacadura, J. F., J. Heat Mass transf. 15 (1972) 1451/65.

[16] Pateyron, B., Adissin, A., Martin, C., Fauchais, P., Revue Gén. Therm. 172 (1976) 313/25.

[17] Porteus, J. O., J. Opt. Soc. Am. 53 (1963) 1394.

[18] Price, D. J., Proc. Phys. Soc. 59 (1947) 118/31.

[19] ThoRn, J., Winslon, G. H., Temperature its measurement and control radiation of thermal energy from real bodies. Ed. F. G. Kwedde (Reinhold publ. Co, New York) 1962, 421/47.

[20] Ward, L., Proc. Phys. Soc. 369 (1956) 339/43. 